\title{
Refractory Bergmann type A bile leak: the need to strike a balance
}

\section{(ㄷ)(1)}

\author{
Authors \\ Giulia Bonato ${ }^{1}$, Alberto Tringali ${ }^{1}$, Lorenzo Dioscoridi ${ }^{1}$ \\ Institutions \\ 1 Digestive and Operative Endoscopy Unit, Niguarda-Ca' \\ Granda Hospital, Milan, Italy \\ 2 Digestive Endoscopy Unit, Fondazione Policlinico \\ Universitario A. Gemelli, IRCCS, Rome, Italy \\ 3 Endoscopy Department, St Lukes Private Hospital, \\ Panorama, Thessaloniki, Greece
}

Massimiliano Mutignani ${ }^{1}$, Edoardo Forti ${ }^{1}$, Alberto Larghi ${ }^{2}$, Stefanos Dokas ${ }^{3}$, Francesco Pugliese ${ }^{1}$, Marcello Cintolo ${ }^{1}$,

submitted 14.3 .2018

accepted after revision 25.7.2018

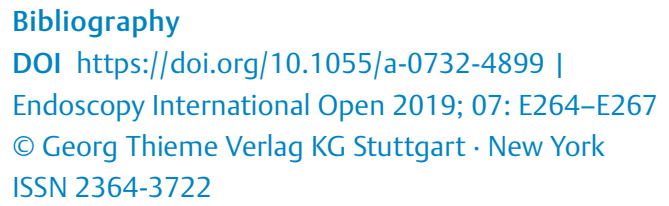

Corresponding author

Massimiliano Mutignani, MD, Digestive and Operative

Endoscopy Unit, Niguarda-Ca' Granda Hospital, Piazza dell'Ospedale Maggiore 3, 20121, Milan, Italy

Fax: +00390264442471

massimiliano.mutignani@ospedaleniguarda.it

\section{ABSTRACT}

Background and study aims Endoscopic therapy for postoperative Bergmann type A bile leaks is based on biliary sphincterotomy \pm stent insertion. However, recurrent or refractory bile leaks can occur.

Patients and methods This was retrospective study including all consecutive patients who were referred to our center with a Bergmann type A bile leak refractory to previous conventional endoscopic treatments.

Results Seventeen patients with post-cholecystectomyrefractory Bergmann type A bile leak were included. All had received prior endoscopic biliary sphincterotomy with biliary stent or nasobiliary catheter placement and all had a percutaneous or surgical abdominal drainage. Repeat endoscopic retrograde cholangiopancreatography (ERCP) confirmed a Bergmann type A bile leak and in all patients we observed that the abdominal drainage was placed adjacent to the origin of the fistula. Our treatment consisted of pulling the drain away from the fistulous site, with extension of the previous sphincterotomy when needed. The treatment was successful in all cases. Mild complications occurred in three patients.

Conclusions Our retrospective study shows that refractory Bergmann type A bile leak may be a consequence of an unfavorable position of the abdominal drainage tube, which can be corrected by pulling the drain away from the origin of the fistula. This establishes a favorable pressure gradient that leads the bile flowing from the bile duct into the duodenum.

\section{Introduction}

Cholecystectomy is one of the most common surgical operations worldwide [1]. Postoperative adverse events are also common and include, amongst others, bile leakage from the cystic stump or from bile ductules just beneath the Gleason's capsule [2]. In these cases, surgical reintervention is associated with high morbidity and severe technical difficulties, especially when performed late [2]. Thus, endoscopic treatment of bile leak from the cystic duct has become the treatment of choice $[2,3]$. Surgical reintervention is associated with high morbidity and severe technical difficulties, especially when performed late [2]. Endotherapy consists of biliary sphincterotomy with the rationale of reducing the pressure gradient between the bile duct and the duodenum [4]. Many authors, however, described failures of this standard treatment and suggested the additional placement of a stent in the common bile duct as an adjunct treatment (using either plastic or self-expandable metal stents) [5-8]. Despite all these treatments, bile leaks refractory to endoscopic treatment can occur, with scanty recommendations on how they should be managed. We report a se- 
- Table 1 Resuming previous and new treatments in our case series of Bergmann type A biliary leaks.

\begin{tabular}{|c|c|c|c|c|}
\hline $\begin{array}{l}\text { Number of new treatments } \\
\text { Number of previous treatments }\end{array}$ & & Drain retrieval & Extension of BS + stent removal & NBD placement \\
\hline $\mathrm{BS}+$ stent placement & 15 & 15 & 15 & 13 \\
\hline $\mathrm{BS}+\mathrm{NBD}$ placement & 2 & 2 & 0 & 0 \\
\hline тот & 17 & 17 & 15 & 13 \\
\hline
\end{tabular}

ries of "refractory" Bergmann type A bile leaks that were treated based on gradient pressure mechanisms.

\section{Patients and methods}

Data on all consecutive patients who were referred to our center from outside hospitals with a post-colecystectomy bile leak refractory to conventional endoscopic treatments between November 2010 to November 2015 were retrospectively retrieved from our prospectively collected institutional database. Only patients with a Bergmann type A bile leak, defined as biliary leakage from the cystic duct or peripheral hepatic radicals, were included in this case series. The diagnosis of Bergmann type A leak was based on an occlusion cholangiogram performed during endoscopic retrograde cholangiopancreatography (ERCP) in the other hospitals, which was confirmed during ERCP in our center. Patients all had undergone previous ERCP with sphincterotomy with stent or nasobiliary drainage placement and all had an abdominal drainage tube placed during surgery of by interventional radiologists. The study was approved by our institutional review board and written informed consent was obtained from patients before all procedures described.

\section{Results}

During the study period, 17 patients (12 females, 5 males; mean age: $53 \pm 9$ years) referred to our unit because of refractory, postoperative bile leak were diagnosed with a refractory Bergmann type A leak. All fistulas occurred after cholecystectomy (15 laparoscopic, two open). All patients had received treatment in various outside hospitals ( $\triangleright$ Table 1 ). Twelve patients were treated by insertion of a percutaneous drainage when bile leak occurred and the remaining five patients already had a surgical drain placed (in two cases placed after secondlook laparotomy). In addition, all patients underwent ERCP with biliary sphincterotomy and insertion of a plastic biliary stent $(n=15)$ or nasobiliary drainage $(n=2)$.

Mean outflow of the fistula was $530 \mathrm{~mL} /$ day (range: 80 $1400 \mathrm{~mL}$ ). Fifteen patients had a high-output bile fistula (>200 mL/day), while two patients presented with a low-output fistula (<200 mL/day). In 14 patients, amylase levels were measured from the drainage fluid, which in 12 cases were $>500 \mathrm{UI} / \mathrm{L}$ positive values were observed (>500 UI/L) probably because of reflux of duodenal content through the stent. In the remaining two cases, amylase levels were within normal range. In our unit, a diagnostic balloon occlusion cholangiogram was first performed during ERCP to verify the integrity of the biliary tree and localize the site of the leak. In 12 cases, the origin of the leak was from the cystic stump; in four cases from small subcapsular branches at the level of the gallbladder fossa; and in the remaining one case from cholecystic stump (after subtotal cholecystectomy). In all patients, we observed at fluoroscopy that the abdominal percutaneous/surgical drainage tube was located just adjacent to the origin of the fistula. Inspired by this observation, in the two patients with nasobiliary drainage, an attempt was made to resolve the fistula by pulling back the abdominal drain 3 to $4 \mathrm{~cm}$ away from the origin of the fistula and was successful in both cases. Following these experiences, in the remaining 15 patients, we developed a therapeutic approach as follow: in those with previously placed stent, removal of the stent and extension of the previous biliary sphincterotomy was done when necessary, which was then followed by pulling the abdominal drainage tube back 3 to $4 \mathrm{~cm}$ as in the first two cases. In 13 patients, a 7Fr nasobiliary drainage was also placed, while in the two patients with low-output fistula, no other treatments were performed. In all cases, resolution of the bile leak was obtained.

The time for healing of the fistula was 24 hours in 14 cases, 48 hours in two cases and 72 hours in another case. When the fistula output stopped, after confirmation by abdominal ultrasound or computer tomography, the abdominal drainage tube was removed. Three patients experienced mild complications, such as bleeding during extension of the biliary sphincterotomy, which was successfully treated by adrenalin injection and two cases of self-limited fever within the first postprocedural day, probably due to transient bacteremia. All patients were discharged within 1 week (range: 3-7 days). Follow-up lasted 6 months during which no complication or recurrence of the leak was observed.

\section{Discussion}

We presented our experience in treating patients with postcholecystectomy refractory Bergmann type A biliary leak who had failed previous conventional endoscopic treatments, performed in outside hospitals. In all patients we observed, at fluoroscopy, that the abdominal percutaneous/surgical drainage tube was located just adjacent to the origin of the fistula, which led us to theorize that the first therapeutic maneuver 
could have been to pull back the drainage tube few centimeters away from the fistula to change the pressure dynamics around the site of the bile leak.

Post-cholecystectomy bile leak is not an uncommon adverse event (AE) and can occur in as much as $1 \%$ of the cases [2]. The leak is usually easily recognized as the abdominal drain contains bilious fluid. Leaks are classified according to volume (low- or high-output fistulae) and location. There are numerous anatomic classifications of postoperative bile leaks/injuries. The Bergmann classification is a general and concise classification of bile duct injuries during laparoscopic cholecystectomy [5]. The simplest bile duct injury in the Bergmann classification is type A injury, which is a bile leak originating from the cystic stump or from the small radicals at the level of the gallbladder fossa. This type of $A E$ is the most common and easiest to treat. Endoscopic intervention represents the treatment of choice of Bergmann type A bile leaks. ERCP with cholangiogram is first performed to confirm localization of the leak and is followed by biliary sphincterotomy, while placement of a biliary stent or a nasobiliary drainage is usually not necessary. However, stenting is recommended to ensure adequate biliary drainage in cases of incomplete sphincterotomy or retained stone. The aim of the endoscopic treatment is to decrease pressure at the level of the biliary sphincter, which creates a favorable pressure gradient for the bile that flows from the duct through the sphincterotomy into the duodenum.

With the biliary sphincter intact, intrabiliary pressure (mean value $\sim 20 \mathrm{~cm} \mathrm{H}_{2} \mathrm{O}$ ) is considerably higher than the intrabdominal pressure (mean value $\sim 10 \mathrm{~cm} \mathrm{H}_{2} \mathrm{O}$ ) [6]. The presence of an abdominal drain drastically modifies the intrabdominal pressure by creating an intraabdominal pressure almost equal to the atmospheric pressure (conventionally considered as $0 \mathrm{~cm}$ $\mathrm{H}_{2} \mathrm{O}$ ), at the level of the drain and of the bile leak. As a consequence of this pressure balance, the bile moves from the bile duct into the abdominal cavity and outside through the drain ( $\triangleright$ Fig.1). On the other hand, if a surgical drain is not present, the bile would leak from the open cystic stump into the abdomen, thus favoring formation of a bile collection at the level of the subhepatic space, close to the site of cholecystectomy. To promote fistula healing, diversion of the bile flow from the bile duct into the duodenum should be obtained. For Bergmann type A fistulas, this goal is achieved by performing biliary sphincterotomy, which can be associated with removal of bile duct stones if present.

In our retrospective study in patients with a refractory Bergmann type A fistula, despite failure of previous endoscopic interventions, $100 \%$ treatment success was obtained by simply moving the abdominal drain away from the origin of the fistula. By doing this maneuver, we eliminated the low atmospheric pressure, allowing restoration of the favorable pressure gradient that moves the bile flow toward the duodenum ( $\triangleright$ Fig.2). In addition, there will be flux of intestinal fluid into the abdominal cavity, as we encountered in many of our cases, which is not sterile and can cause serious complications.

Along with biliary sphincterotomy, many authors proposed bile duct stent placement to treat refractory biliary leaks after cholecystectomy. For this purpose, Rustagi et al. used both

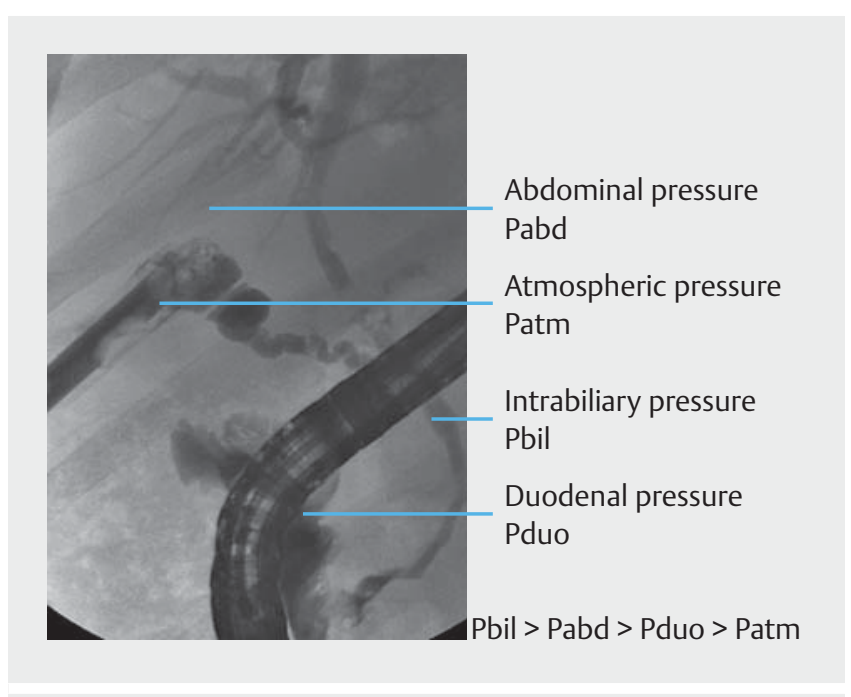

Fig. 1 Pressure balance at the site of a Bergmann type A bile leak.

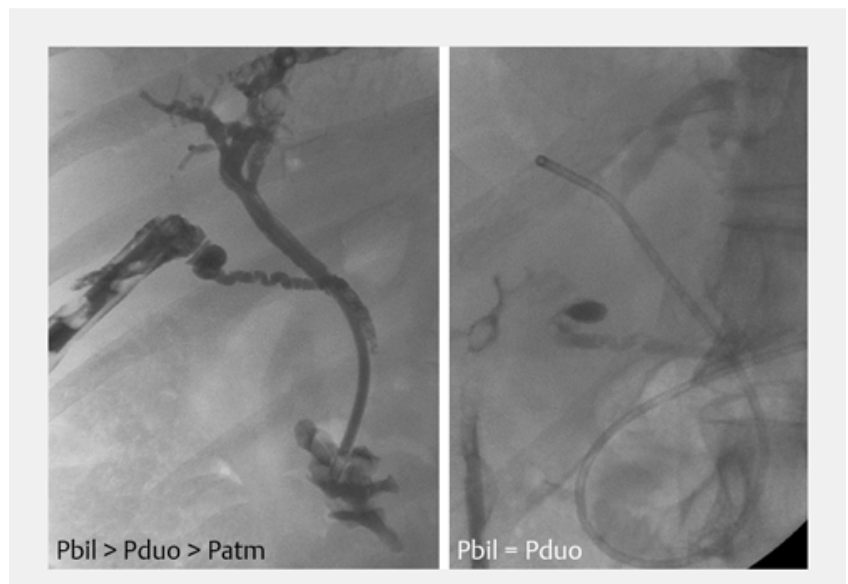

- Fig. 2 New pressure balance after our endotherapy in case of "refractory" Bergmann type A bile leak.

plastic and fully-covered metal stents in 20 patients [7]. Adler et al. presented the results of a large series of 518 patients treated endoscopically for traumatic or postsurgical bile duct leaks. Although the most common site of bile leak was the cystic duct stump, they always inserted a plastic or fully-covered metal stent [8]. Martin et al. described four patients with cystic stump dehiscence who were treated by placement of a fullycovered metal stent [9]. Finally, Ibrarullah et al. reported their management of bile duct injuries at various stages of presentation. In case of bile leakage with intact common bile duct, an endoscopic sphincterotomy with insertion of a $10 \mathrm{Fr}$ plastic stent was always performed [10]. If the biliary plastic stent is placed close to the origin of the leak, this may cause contamination of the abdominal cavity as mentioned earlier. Thus, caution should be taken to choose the stent with the appropriate length to avoid occurrence of this complication. On the other hand, fully-covered metal stents are obviously effective because they seal the leak. However, they are very expensive and 
their use is not justified by any cost-benefit analysis. Moreover, a repeat ERCP has to be performed to remove any kind of stent.

In our opinion, biliary stents should be reserved for particular cases, i. e. incomplete biliary sphincterotomy, extrinsic compression/stenosis of the common bile duct or remnant bile duct stones. In our series, we extended incomplete sphincterotomies to guarantee a "therapeutic" pressure gradient without biliary stenting. In 13 cases, we left a nasobiliary drain in place to help confirm correct healing of the fistula, without any therapeutic intent.

Our treatment is easily reproducible and can be offered as a remote consultation after discussing radiological images and clinical status of the patient. The latter can be of value in reducing expenses involved with patient relocation and additional interventions in other hospitals. During the last year, we provided counseling and successfully treatment remotely in four additional patients from outside hospitals.

\section{Conclusion}

In conclusion, Bergmann type A bile leak can be due to incorrect placement of the abdominal drainage in too close proximity with the site of bile leak, where the very low atmospheric pressure neutralizes the effect of prior endoscopic treatments, such as biliary sphincterotomy and/or stent insertion. By pulling the drain away just a few centimeters, establishment of a favorable pressure gradient can be achieved so that the bile can flow from the bile duct into the intestine, thus resolving the bile leak. This is the way to strike he correct pressure balance!

\section{References}

[1] Amato L, Fusco D, Acampora A et al. Volume and health outcomes: evidence from systematic reviews and from evaluation of Italian hospital data. Epidemiol Prev 2017; 41: 1 -128

[2] Yabe S, Kato H, Mizukawa S et al. Predictive factors for outcomes of patients undergoing endoscopic therapy for bile leak after hepatobiliary surgery. Dig Endosc 2016; 9: 353-361

[3] Mutignani M, Shah SK, Tringali A et al. Endoscopic therapy for biliary leaks from aberrant right hepatic ducts severed during cholecystectomy. Gastrointest Endosc 2002; 55: 932 -936

[4] Kook HK, Tae NK. Endoscopic management of bile leakage after cholecystectomy: a single center experience for 12 years. Clin Endosc 2014; 47: $248-253$

[5] Bergmann J], van den Brink GR, Rauws EA et al. Treatment of bile duct lesions after laparoscopic cholecystectomy. Gut 1996; 38: 141 - 147

[6] Guyton AC, Hall JE. Fisiologia Medica. XII edition. Edra Ed 2011

[7] Rustagi T, Aslanian HR. Endoscopic management of biliary leaks after laparoscopic cholecystectomy. J Clin Gastroent 2014; 48: 674-678

[8] Adler DG, Papachristou GI, Taylor L] et al. Clinical outcomes in patients with bile leaks treated via ERCP with regard to the timing of ERCP: a large multicenter study. Gastrointest Endosc 2017; 85: 766 772

[9] Martin D, Uldry E, Demartines N et al. Bile duct injuries after laparoscopic cholecystectomy: 11-year experience in a tertiary center. BioScience Trends 2016; 10: 197 - 201

[10] Ibrarullah M, Sankar S, Sreenivasan K et al. Management of bile duct injury at various stages of presentation: experience from a tertiary care centre. Indian J Surg 2015; 77: $92-98$

\section{Competing interests}

\section{None}

